\title{
Detection of RACK1 and CTNNBL1-induced activation of mouse splenocytes using an immunoprecipitation-based technique
}

\author{
BOHAN DONG ${ }^{1,2}$, GUANGLI DAI ${ }^{3}$, LEI XU $^{1,2}$ and DAMIN SHI ${ }^{1}$ \\ ${ }^{1}$ Department of Biochemistry; ${ }^{2}$ Anhui Key Laboratory of Active Biological Macro-Molecules, \\ Wannan Medical College, Wuhu, Anhui 241002; ${ }^{3}$ Department of Gynecology and Obstetrics, \\ Traditional Chinese Medical Hospital of Wuhu, Wuhu, Anhui 241000, P.R. China
}

Received November 17, 2016; Accepted July 4, 2017

DOI: $10.3892 / \mathrm{mmr} .2017 .7485$

\begin{abstract}
Tumor cell lysates (TCLs) have been reported to induce antitumor immunity; however, it remains unclear which elements serve a role in this process. The present study identified 768 proteins that were upregulated in TCL prepared from Lewis lung cancer cells compared with the lysate from type II alveolar epithelial cells. Among the proteins that were upregulated in TCL, receptor for activated $\mathrm{C}$ kinase 1 (RACK1) and catenin $\beta$-like 1 (CTNNBL1) are closely associated with cell proliferation and the inhibition of apoptosis. To determine the role of these proteins in TCL, a protein extraction method was designed, which was based on immunoprecipitation. Using this method, RACK1 and CTNNBL1 were extracted, whereas the other proteins within the TCL were not affected. The modified TCL exhibited a stronger ability to induce splenocyte apoptosis, whereas the ability to promote cell activation was reduced. These findings suggested that the TCL depends on RACK1 and CTNNBL1 to activate mouse immunocytes, including monocytes and B lymphocytes, and inhibit apoptosis. Therefore, the present study may provide information regarding the composition of TCLs and their positive regulatory effect on immunocytes.
\end{abstract}

\section{Introduction}

Tumor incidence is associated with impaired antitumor immunity in the patients. Effectively stimulating the immune system to kill tumor cells is a critical factor for the success or failure of antitumor immunotherapy (1). Efforts have been made to construct antitumor vaccines, aiming to induce antitumor immunity using tumor cells, genes that express tumor antigens

Correspondence to: Dr Bohan Dong, Department of Biochemistry, Wannan Medical College, 22 Wenchang West Road, Wuhu, Anhui 241002, P.R. China

E-mail: 240151563@qq.com

Key words: tumor cell lysate, vaccine, mouse splenocytes, immunocyte, apoptosis, lung cancer, receptor for activated $\mathrm{C}$ kinase 1 , catenin $\beta$-like 1 or tumor-associated proteins and peptides (2). Tumor cell lysates (TCLs) have been reported to induce antitumor immunity, since all antigens and signal transduction factors that are present in tumor cells exist in the TCL $(3,4)$; thus, they may have potential to be used in antitumor vaccines. In a dendritic cell (DC)-based antitumor study, lung cancer TCL was used to activate mouse DCs in vitro; subsequently, the activated DCs were transfused back into the mice to treat the tumor (5). In addition, our previous study combined Lewis lung cancer TCL with heat shock protein 65 to activate immunocytes in mice; the results demonstrated that the activated immunocytes induced anti-lung cancer immunity in vivo (6). Although TCLs have been used in antitumor immunotherapy, the key proteins involved in antitumor immunity remain unknown. Receptor for activated $\mathrm{C}$ kinase 1 (RACK1) is expressed in numerous tumor types, including lung cancer, colorectal cancer and hepatocellular carcinoma (7). This factor is able to activate the Sonic hedgehog signaling pathway, which promotes tumor cell proliferation and inhibits apoptosis (8). Similarly, catenin $\beta$-like 1 (CTNNBL1) is a protein that has been detected in colorectal cancer, osteosarcoma and B lymphocytes (9-12). Although the function of this protein has yet to be determined, it has been identified as a putative regulator of the canonical Wnt signaling pathway using RNA interference technology, where it acts upstream of, or in parallel to $\beta$-catenin, particularly in colorectal cancer cell proliferation (9). RACK1 and CTNNBL1 are expressed in numerous tumor cell types to transduce the Sonic hedgehog cell proliferation signaling pathway; therefore, it is hypothesized that these proteins must also exist in TCLs. In the present study, RACK1 and CTNNBL1 were detected in TCL prepared from Lewis lung cancer cells, and their roles in the activation of mouse splenocytes were determined.

\section{Materials and methods}

Animals and cell lines. Female C57BL/6 mice ( $\mathrm{n}=9$; age, 6-8 weeks; weight, 18-22 g) were purchased from Anhui Medical University Laboratory Animal Center (Hefei, China) and were maintained in microisolator cages under pathogen-free conditions, at a temperature of $20-26^{\circ} \mathrm{C}$ under a 12-h light/dark cycle. Food and water was autoclaved before feeding and provided ad libitum. The mice were evenly 
divided into 3 groups; a PBS group, whole TCL group and TCL RACK1-/CTNNBL1-group. Experimental manipulation of the mice was undertaken in accordance with the National Institutes of Health Guide for the Care and Use of Laboratory Animals (https://www.ncbi.nlm.nih.gov/books/NBK54050), with the approval of the Scientific Investigation Board of Science and Technology of Anhui Province and the animal experiments of the present study were also approved by the Ethics Committee of Wannan Medical College. The mouse Lewis lung cancer cell line was purchased from Wuhan Boster Biological Technology, Ltd. (Wuhan, China). The cells were cultured in high-glucose Dulbecco's modified Eagle's medium (Hyclone; GE Healthcare Life Sciences, Logan, UT, USA) supplemented with $10 \%$ fetal calf serum (FCS; Gibco; Thermo Fisher Scientific, Inc., Waltham, MA, USA), 100 U/ml penicillin and $100 \mu \mathrm{g} / \mathrm{ml}$ streptomycin (Sigma-Aldrich; Merck KGaA, Darmstadt, Germany). The present study was approved by the Ethics Committee of Wannan Medical College (Wuhu, China).

Preparation of TCL. To prepare the TCL, cultured Lewis cells were lysed using a freeze-thaw cycle in PBS solution between $-70^{\circ} \mathrm{C}$ and $37^{\circ} \mathrm{C}$. After five cycles, the prepared TCL was stored at $-70^{\circ} \mathrm{C}$ until further use. The TCL was observed under a microscope (Olympus Corporation, Tokyo, Japan) using trypan blue staining (Sigma-Aldrich; Merck KGaA) to ensure all of the cells were lysed.

Preparation of mouse type II alveolar epithelial cell lysate. To prepare the lysate, cultured type II alveolar epithelial cells were lysed using a freeze-thaw cycle in PBS solution between $-70^{\circ} \mathrm{C}$ and $37^{\circ} \mathrm{C}$. After five cycles, the prepared lysate was stored at $-70^{\circ} \mathrm{C}$ until further use. The lysate was observed under a microscope (Olympus Corporation, Tokyo, Japan) using trypan blue staining (Sigma-Aldrich; Merck KGaA) to ensure all of the cells were lysed.

Isobaric tags for relative and absolute quantitation (iTRAQ) analysis. TCL was prepared from Lewis lung cancer cells or mouse type II alveolar epithelial cell using the above method. Protein concentration of TCL was determined using a bicinchoninic acid (BCA) assay using bovine serum albumin as standard (BCA Assay kit; Beyotime Institute of Biotechnology, Shanghai, China). Subsequently, $100 \mu \mathrm{g}$ TCL protein was digested with trypsin (Promega Corporation, Madison, WI, USA) overnight at $37^{\circ} \mathrm{C}$. Digested samples were labeled using the 8-plex iTRAQ kit (Applied Biosystems; Thermo Fisher Scientific Inc.) according to the manufacturer's protocol. iTRAQ-labeled samples were diluted to $100 \mu \mathrm{l}$ with $20 \mathrm{mM} \mathrm{HCOONH}_{4}, 2 \mathrm{M} \mathrm{NaOH}$ (pH 10) prior to high-performance liquid chromatography on a Gemini-NX 3u C18 110A (150x2.00 mm) column (Phenomenex, Torrance, CA, USA). Peptides were separated using linear gradient elution; the mobile phases were comprised as follows: Mobile phase A, $20 \mathrm{mM} \mathrm{HCOONH}_{4}$ and $2 \mathrm{M} \mathrm{NaOH}$; mobile phase $\mathrm{B}$, $20 \mathrm{mM} \mathrm{HCOONH}_{4}, 2 \mathrm{M} \mathrm{NaOH}$ and $80 \%$ acetonitrile (ACN). The gradient was increased from 5 to $40 \%$ mobile phase B in $30 \mathrm{~min}$ at a flow rate of $0.2 \mathrm{ml} / \mathrm{min}$. The UV detector was set at $214 / 280 \mathrm{~nm}$, and fractions were collected every $1 \mathrm{~min}$. In total, 24 fractions were collected and dried by vacuum centrifuge in
$12,000 \times \mathrm{g}$ overnight at $4^{\circ} \mathrm{C}$. Acidified with $50 \% \mathrm{CF}_{3} \mathrm{COOH}$, the peptides were separated using linear gradient elution; the mobile phases were comprised as follows: Mobile phase A, $5 \% \mathrm{ACN}$ and $0.1 \%$ formic acid (FA); mobile phase B, $80 \%$ $\mathrm{ACN}$ and $0.1 \%$ FA. The gradient was increased from 5 to $90 \%$ mobile phase $\mathrm{B}$ in $50 \mathrm{~min}$ at a flow rate of $300 \mathrm{nl} / \mathrm{min}$. The tandem mass spectrometry (MS/MS) analysis was performed using a Q Exative system (Thermo Fisher Scientific, Inc.) in Information-Dependent Mode. Briefly, MS spectra were acquired across the mass range of $350-1800 \mathrm{~m} / \mathrm{z}$ in high resolution mode $(>70,000$ full width at half maximum) using a $40 \mathrm{msec}$ accumulation time per spectrum. A maximum of 20 precursors per cycle were chosen for fragmentation from each MS spectrum with $60 \mathrm{msec}$ minimum accumulation time for each precursor and dynamic exclusion for $20 \mathrm{sec}$. Tandem mass spectra were recorded in high sensitivity mode (resolution $>17,500$ ) with rolling collision energy on and iTRAQ reagent collision energy adjustment on. For protein identification, the MS/MS spectra were processed using ProteinPilot software version 5.0 (Sciex, Framingham, MA, USA). Differentially abundant proteins were examined using QuickGO (https://www.ebi.ac.uk/QuickGO/) for Gene Ontology annotation and enrichment analysis.

Reverse transcription-quantitative polymerase chain reaction $(R T-q P C R)$. Total RNA was extracted from mouse Lewis lung cancer cell whole TCL and reverse transcribed into cDNA using an RNAprep pure kit (Tiangen Biotech Co. Ltd., Beijing, China) and FastQuant RT kit (with gDNase; Tiangen Biotech Co. Ltd.), respectively. Oligo dT mixed with total RNA was and placed in a warm bath at $70^{\circ} \mathrm{C}$ for $10 \mathrm{~min}$, and then into ice-water mixture. $d N T P \backslash R T a s e \backslash R$ nase free $\mathrm{H}_{2} \mathrm{O}$ was added to prepare the reverse transcription reaction system. The system was warmed at $42^{\circ} \mathrm{C}$ for $1 \mathrm{~h}$, and then was heated to $70^{\circ} \mathrm{C}$ for10 min to produce cDNA. Subsequently, qPCR was performed to detect the mRNA expression levels of RACK1, CTNNBL1, Cullin 3, B-cell lymphoma 2-associated transcription factor 1 (BCLAF1), ribosomal protein S6 (RPS6), superoxide dismutase 1 (SOD1), high mobility group box 1 (HMGB1), inhibitor of growth family member 1 (ING1), ERCC excision repair 3, TFIIH core complex helicase subunit (ERCC3) and GAPDH in mouse Lewis lung cancer cell TCL using a SuperReal PreMix Plus kit with SYBR Green (Tiangen Biotech Co. Ltd.). Thermocycling conditions were $30 \mathrm{sec}$ at $95^{\circ} \mathrm{C}, 2$ step PCR at $95^{\circ} \mathrm{C}$ for $0.5 \mathrm{sec}$ and at $60^{\circ} \mathrm{C}$ for $30 \mathrm{sec}$, for 40 cycles. Following dissociation was 1 cycle at $95^{\circ} \mathrm{C}$ for $15 \mathrm{sec}$, at $60^{\circ} \mathrm{C}$ for $30 \mathrm{sec}$ and at $95^{\circ} \mathrm{C}$ for $15 \mathrm{sec}$ successively. The qPCR data were analyzed with the ABI Step One PCR system (Applied Biosystems; Thermo Fisher Scientific Inc.). Relative gene expression was quantified using the $2^{-\Delta \Delta \mathrm{Cq}}(13)$ and normalized to GAPDH (14). All qPCR primer sequences are presented in Table I.

Immunoprecipitation (IP). Protein concentration of the TCL prepared from Lewis lung cancer cells was measured using the BCA protein assay kit. Subsequently, IP was conducted using a Pierce Classic IP kit (cat. no. 26146; Pierce; Thermo Fisher Scientific, Inc.). For $1 \mathrm{mg}$ lysate, $80 \mu \mathrm{l}$ Control Agarose Resin slurry was added to preclear the lysate. Subsequently, anti-RACK1 (cat. no. 5432; dilution, 1:50; Cell Signaling 
Table I. Primer sequences for quantitative polymerase chain reaction.

\begin{tabular}{lll}
\hline Gene & \multicolumn{1}{c}{ Forward primer $\left(5^{\prime}-3^{\prime}\right)$} & Reverse primer $\left(5^{\prime}-3^{\prime}\right)$ \\
\hline BCLAF1 & TGAGACGACCTTATGGGTACA & ATCTGCTTCGGGATCTTTGAG \\
RPS6 & TGTTACTCCTCGTGTCCTGC & CCAAAAGTTAGCGTATTCTGC \\
SOD1 & TGTACCAGTGCAGGACCTCAT & GCCCAAGTCATCTTGTTTCTC \\
HMGB1 & GCAGATGACAAGCAGCCCTAT & GCTCTTTCAGCCTTGACCAC \\
ING1 & CAGGCAGATAAGCCGAATAAC & AGGAGACCTGGTTGCACAGAC \\
ERCC3 & CCCGATGTCTCCCGAGTTCTA & GGTGTTGATTTTGGGGTTGTG \\
CTNNBL1 & TTGGCTTACGGACCATCTTTC & TGTTTCTCCCCTTCAATCTTCT \\
RACK1 & TGAAGCAAGAAGTTATCAGCAC & CTCGCACCAAGTTGTCTGTAT \\
GAPDH & TGGTGAAGGTCGGTGTGAAC & GCTCCTGGAAGATGGTGATGG
\end{tabular}

BCLAF1, B-cell lymphoma 2-associated transcription factor 1; CTNNBL1, catenin $\beta$-like 1; ERCC3, ERCC excision repair 3; HMGB1, high mobility group box 1; ING1, inhibitor of growth family member 1; RACK1, receptor for activated C kinase 1; RPS6, ribosomal protein S6; SOD1, superoxide dismutase 1 .

Technology, Inc., Danvers, MA, USA) was added to the precleared cell lysate in a microcentrifuge tube; the antibody/lysate solution was diluted to $300-600 \mu 1$ with PBS. The solution was incubated overnight at $4^{\circ} \mathrm{C}$ to form the immune complex in the spin column. To capture the immune complex, the antibody/lysate sample was added to Protein A/G Plus Agarose in the spin column (supplied in the IP kit), and the column was incubated with gentle end-over-end shaking for $1 \mathrm{~h}$ at $4^{\circ} \mathrm{C}$. Subsequently, the sample was centrifuged $100 \mathrm{xg}$ for $1 \mathrm{~min}$ and the flow-through was saved. The spin column containing the resin was transferred into a new collection tube and $2 \mathrm{X}$ reducing sample buffer was added and incubated at $100^{\circ} \mathrm{C}$ for $5-10 \mathrm{~min}$. The samples were further centrifuged to collect the eluate. Finally, the eluate and flow-through were separated by $10 \%$ SDS-PAGE and analyzed by western blotting. First round IP can remove RACK1 from the TCL. To remove CTNNBL1, the flow-through of first round IP was collected and then used to process second round of IP with anti-CTNNBL1 at $5 \mu \mathrm{g} / \mathrm{mg}$ of lysate (cat. no. ab95170; Abcam, Cambridge, MA, USA). Besides antibody, other steps of second round of IP is the same as the first round.

Flow cytometric analysis. C57/BL6 mice (3 per group) were used for spleen isolation. The spleen was grinded, and depleted of red blood cells using a red blood cell lysis buffer (Tiangen Biotech Co. Ltd.). Then, the spleen cells were centrifuged at $500 \mathrm{x}$ g for $5 \mathrm{~min}$ at room temperature, counted and cocultured at a density of $1 \times 10^{6} / \mathrm{ml}$ with either TCL prepared from Lewis lung cancer cells $\left(1 \times 10^{6}\right)$, TCL with RACK1 and CTNNBL1 removed or PBS for $48 \mathrm{~h}$ at $37^{\circ} \mathrm{C}$. Subsequently, the cells were collected, washed and resuspended (1,000 cells) in PBS supplemented with $1 \%$ heat-inactivated FCS. Thereafter, the mouse spleen cells were stained at room temperature for $30 \mathrm{~min}$ with Annexin V-fluorescein isothiocyanate (FITC)/propidium iodide apoptosis detection kit (cat. no. KGA107; Nanjing KeyGen Biotech Co., Ltd., Nanjing, China) to analyze apoptosis, or with FITC-labeled anti-cluster of differentiation (CD)69 (cat. no. 11-0691-81; eBioscience; Thermo Fisher Scientific, Inc.) to detect activation of spleen cells. The cells were then stored at $4^{\circ} \mathrm{C}$ for $30 \mathrm{~min}$, washed with $1 \mathrm{X}$ PBS and analyzed by flow cytometry (FACSCalibur; BD Biosciences, San Jose, CA, USA) using FlowJo version is 7.6 (FlowJo LLC, Ashland, OR, USA). For flow cytometric detection, lymphocytes and monocytes were gated, and $\leq 1 \times 10^{6}$ splenocytes were acquired per test.

Western blot analysis. TCL was prepared from Lewis cells $\left(1 \times 10^{6}\right)$ and was separated by $10 \%$ SDS-PAGE at $20 \mu \mathrm{g}$ per lane. The proteins were then transferred onto a polyvinylidene fluoride membrane. The membrane was blocked with $5 \%$ evaporated milk for $1 \mathrm{~h}$ at room temperature and was then incubated overnight at $4^{\circ} \mathrm{C}$ with anti-RACK1 (cat. no. 5432; 1:1,000; Cell Signaling Technology, Inc., Danvers, MA, USA), anti-CTNNBL1 (cat.no. ab95170; 1:1,000; Abcam, Cambridge, MA, USA), anti-Cullin 3 (cat. no. ab108407; 1:1,000; Abcam) or anti-GAPDH monoclonal antibodies (cat. no. 5174; 1:1,000; Cell Signaling Technology, Inc., Danvers, MA, USA). The membrane was then incubated for $1.5 \mathrm{~h}$ with IRDye $800 \mathrm{CW}$ goat anti-mouse secondary antibody (cat. no. P/N 925-32211; 1:10,000; LI-COR Biosciences, Lincoln, NE, USA) at room temperature. To visualize the protein on the membrane, the membrane was scanned and analyzed using the Odyssey fluorescent scanning system (LI-COR Biosciences).

Statistical analysis. Data are presented as the mean \pm standard deviation of 3 independent experiments. Statistical significance was determined using $\chi^{2}$ test. $\mathrm{P}<0.05$ was considered to indicate a statistically significant difference. Statistical analysis was performed using SPSS software version 22.0 (IBM Corp., Armonk, NY, USA).

\section{Results}

iTRAQ detection of proteins in Lewis lung cancer TCL. Theoretically, all the proteins expressed in the tumor cells should exist in the TCL prepared with from these cells. To determine protein variety and quantitation in TCL, a TCL was prepared from Lewis lung cancer cells and was analyzed using iTRAQ (Fig. 1). Using the iTRAQ detection method, 4,444 confidence proteins were detected in the TCL (Fig. 1C). A 


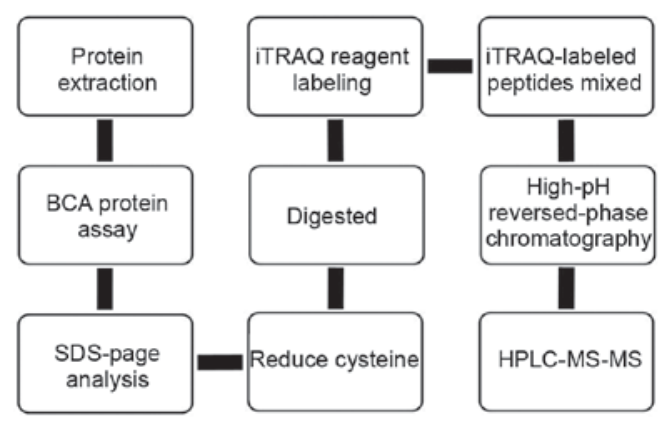

C

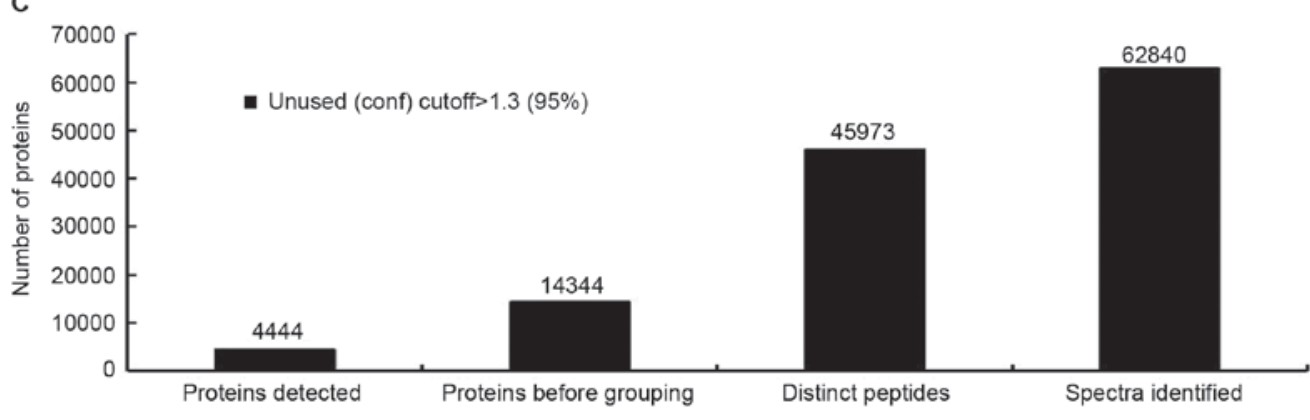

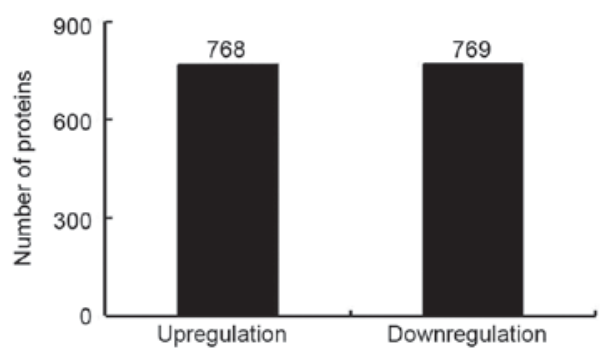

Figure 1. iTRAQ analysis of the proteins in TCL. (A) iTRAQ operation process. (B) Upregulated and downregulated proteins in Lewis lung cancer cell TCL compared with type II alveolar epithelial cell lysate. (C) Proteins, peptides and spectra analyzed by iTRAQ between the Lewis lung cancer cell TCL and type II alveolar epithelial cell lysate. Unused (Conf) Cutoff $>1.3$ (95\%). 'Proteins detected' refers to the number of proteins detected at each confidence cutoff; 'Proteins before grouping' refers to the total number of proteins with a Total ProtScore above the Unused Cutoff; 'Distinct peptides' refers to the number of distinct peptides associated with proteins detected with the specified confidence level or better; 'Spectra identified' refers to the total number of spectra associated with the detected proteins at each threshold. BCA, bicinchoninic acid; HPLC-MS-MS, high-performance liquid chromatography-tandem mass spectrometry; iTRAQ, isobaric tags for relative and absolute quantitation; TCL, tumor cell lysate.

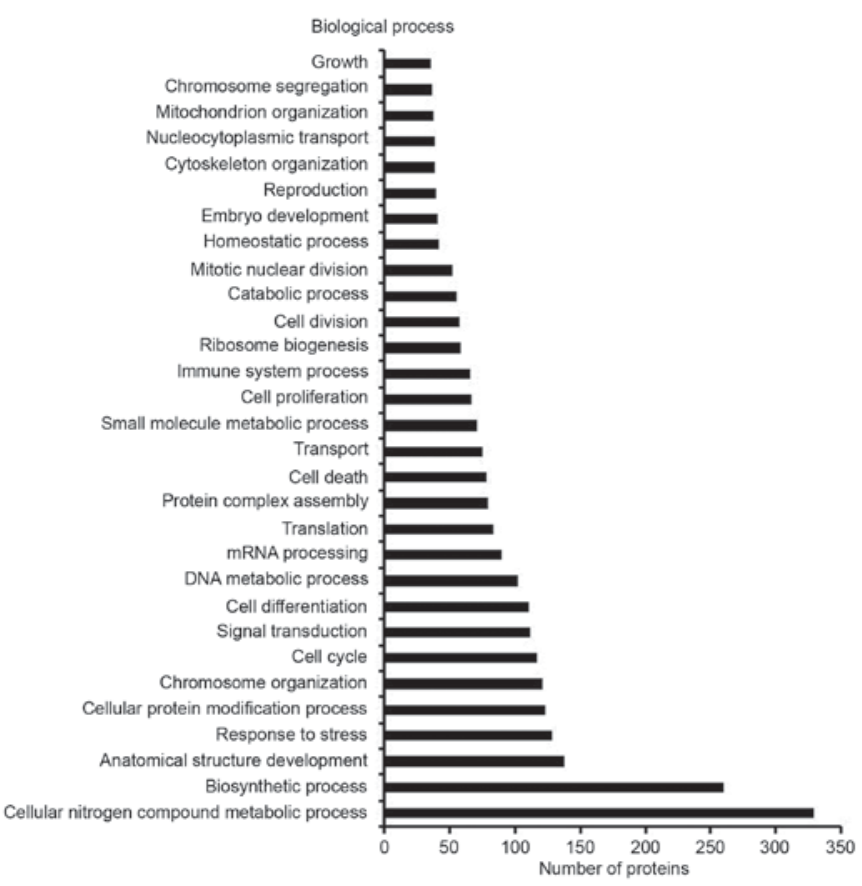

Figure 2. Biological process enrichment analysis of upregulated proteins in Lewis lung cancer cell tumor cell lysate.

confidence protein is one that can be searched in the currently available protein information databases, with high quantitation and structural integrity. Among these proteins, 768 proteins were upregulated in Lewis lung cancer TCL compared with mouse type II alveolar epithelial cell lysate. The Lewis lung cancer TCL/type II alveolar epithelial cell lysate ratio was $>0.67$ (Fig. 1B). A total of 769 proteins were downregulated in the Lewis lung cancer TCL compared with the mouse type II alveolar epithelial cell lysate with a ratio of $>1.5$ (Fig. 1B). These proteins could be classified based on molecular function, cellular component or biological process (Fig. 2), including cell growth, cell division, cell death, cell differentiation, cell proliferation and cell cycle. Among the detected proteins that were differentially expressed between the TCL and the alveolar epithelial cell lysate, $4.56 \%$ were associated with cell growth, $7.42 \%$ were associated with cell division, $10.16 \%$ were associated with cell death, $14.32 \%$ were associated with cell differentiation, $8.72 \%$ were associated with cell proliferation and $15.23 \%$ were associated with cell cycle (Fig. 2). These proteins were selected as the target proteins in the present study, since they serve an important role in cell proliferation and apoptosis.

Target protein screen in Lewis lung cancer TCL. Using the iTRAQ method, a large number of proteins were detected in the TCL that are correlated with cell proliferation. To determine the proteins that have the most influence on cell proliferation and apoptosis, the functions of these proteins were determined using protein databases and previously published literature, and the ratio of protein expression in Lewis lung cancer cell TCL/type II alveolar epithelial cell lysate was analyzed. Subsequently, the following proteins were selected as targets in the present study: RACK1, CTNNBL1, Cullin 3, 
A

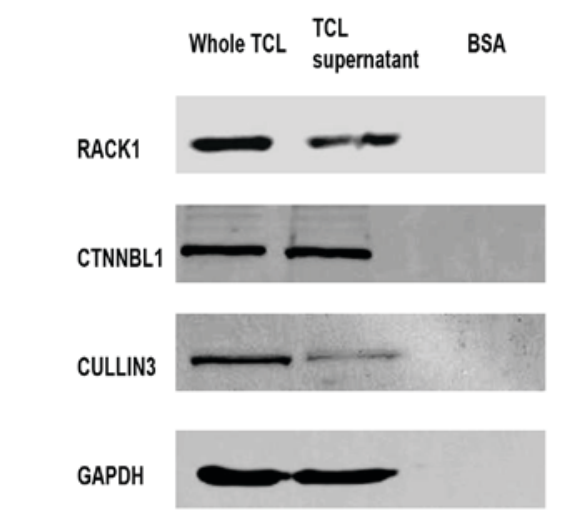

B

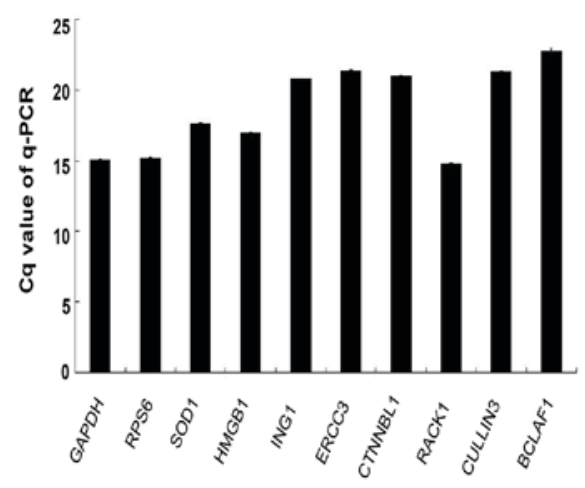

Figure 3. RACK1, CTNNBL1, Cullin 3, BCLAF1, RPS6, SOD1, HMGB1, ING1 and ERCC3 expression in TCL. (A) TCL was prepared from $1 \times 10^{6}$ Lewis lung cancer cells. Immunoblot analysis was performed to detect the protein expression levels of RACK1, CTNNBL1 and Cullin 3 in TCL with specific antibodies. Based on band intensity, RACK1 and CTNNBL1 appeared to be upregulated compared with Cullin 3. (B) Reverse transcription-qPCR was used to detect RACK1, CTNNBL1, Cullin 3, BCLAF1, RPS6, SOD1, HMGB1, ING1 and ERCC3 mRNA expression in $1 \times 10^{6}$ Lewis lung cancer cells. Each histogram represents the mean $\mathrm{Cq}$ value of the PCR products. All genes exhibited increased mRNA expression in Lewis lung cancer cells compared with GAPDH. BSA, bovine serum albumin; BCLAF1, B-cell lymphoma 2-associated transcription factor 1; Cq, quantification cycle; CTNNBL1, catenin $\beta$-like 1; ERCC3, ERCC excision repair 3, TFIIH, core complex helicase subunit; HMGB1, high mobility group box 1; ING1, inhibitor of growth family member 1; qPCR, quantitative polymerase chain reaction; RACK1, receptor for activated C kinase 1; RPS6, ribosomal protein S6; SOD1, superoxide dismutase 1; TCL, tumor cell lysate.

BCLAF1, RPS6, SOD1, HMGB1, ING1 and ERCC3. These proteins have been revealed to promote cell proliferation and inhibit apoptosis in numerous protein databases (Panther, http://pantherdb.org/; UniProt, http://www.uniprot.org/; and NCBI Protein, https://www.ncbi.nlm.nih.gov/protein) and in the literature $(8,9,15-21)$. In addition, these proteins exist in $\geq 2$ signaling pathways that are associated with cell proliferation or apoptosis, and the value of type II alveolar epithelial cell lysate/Lewis lung cancer cell TCL calculated by iTRAQ was $<0.3$ for these proteins. Western blot analysis and RT-qPCR were used to detect the expression levels of these proteins in TCL. The results demonstrated that RACK1, CTNNBL1 and Cullin 3 can be detected by WB, and the expression levels of RACK1 and CTNNBL1 were higher than Cullin 3 in TCL (Fig. 3A). However, BCLAF1, RPS6, SOD1, HMGB1, ING1 and ERCC 3 can only be detected by RT-qPCR, which is known
A

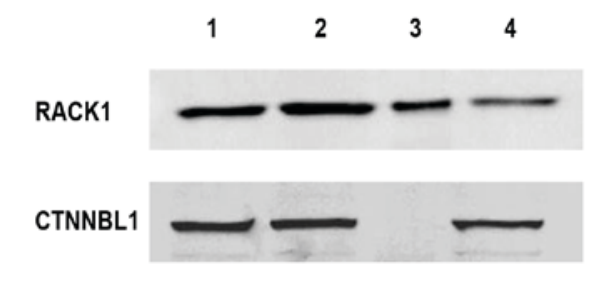

B

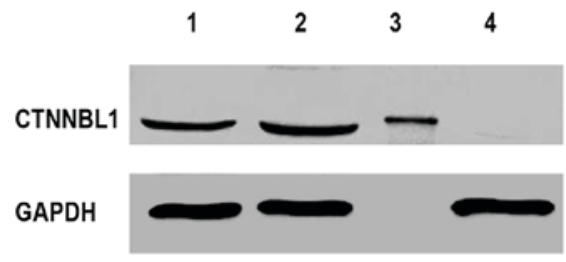

Figure 4. Isolation of RACK1 and CTNNBL1 from TCL. The TCL was prepared from $1 \times 10^{6}$ Lewis lung cancer cells. Subsequently, RACK1 and CTNNBL1 were removed from TCL using IP. Immunoblot analysis was used to detect RACK1, CTNNBL1 and GAPDH expression in whole TCL, TCL supernatant, IP sample or flow-through. (A) First round IP was used to remove RACK1 with an anti-RACK1 antibody. (B) Second round IP was used to remove CTNNBL1 with an anti-CTNNBL1 antibody. Lane 1, TCL supernatant; lane 2, whole TCL; lane 3, IP sample; lane 4, flow-through. CTNNBL1, catenin $\beta$-like 1; IP, immunoprecipitation; RACK1, receptor for activated $\mathrm{C}$ kinase 1; TCL, tumor cell lysate.

to be more sensitive (Fig. 3B). In addition, protein expression was increased in whole TCL compared with the TCL supernatant as determined by western blotting (Fig. 3A). This may be caused by the accumulation of proteins in the lysis cells debris; therefore, the whole TCL was used to investigate its function in subsequent experiments.

Removal of RACK1 and CTNNBL1 from Lewis lung cancer $T C L$. As determined by western blot analysis and RT-qPCR, RACK1 and CTNNBL1 were selected as targets to investigate the function of TCL on mouse splenocytes. The present study used a novel protein extraction method based on IP. To remove both proteins, the method was repeated two times. In the first round, a RACK1 antibody was used to remove RACK1 from the TCL. Following IP, western blotting demonstrated that RACK1 was removed from TCL; however, CTNNBL1 was still detected in the flow-through (Fig. 4A). Similarly, in the second round, CTNNBL1 was removed from the processed TCL after the first round using an anti-CTNNBL1 antibody (Fig. 4B). This resulted in the removal of CTNNBL1 from TCL but not GAPDH; the flow-through of the second round of IP did not contain RACK1 or CTNNBL1; however, other proteins, including GAPDH, were still present. Using two rounds of IP, RACK1 and CTNNBL1 were successfully removed from TCL. The subsequent experiments were performed using TCL that was devoid of RACK1 and CTNNBL1.

Function of RACK1 and CTNNBL1 in mouse splenocytes. To investigate the function of RACK1 and CTNNBL1 in splenocyte activation and apoptosis, mouse splenocytes were incubated with TCL lacking RACK1 and CTNNBL1, and were then analyzed by flow cytometry. The results demonstrated that the expression of the early apoptosis marker Annexin V was higher in mouse splenocytes stimulated with whole TCL 
A

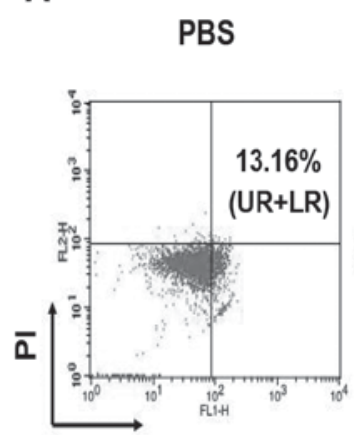

Annexin V

B
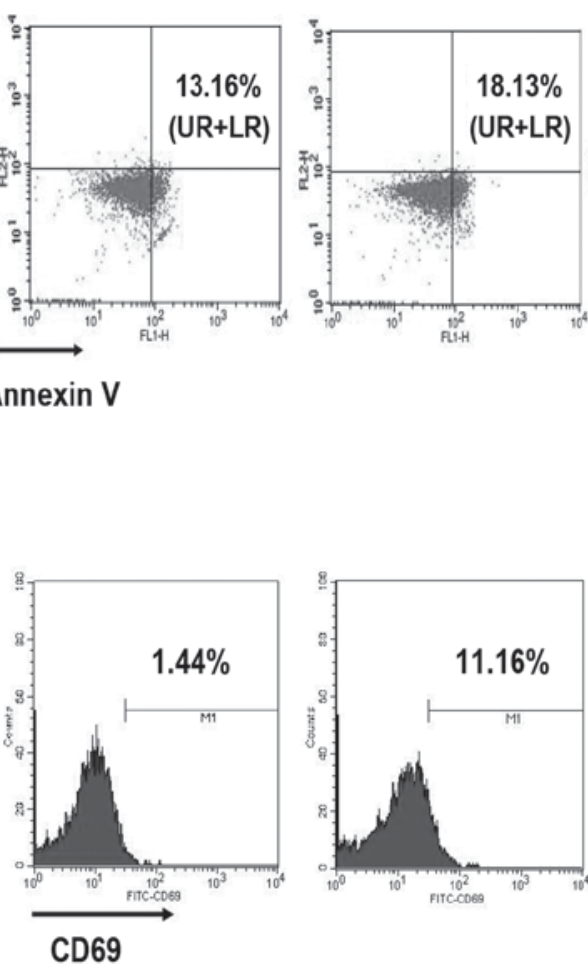

B
TCL RACK1ICTNNBL1.
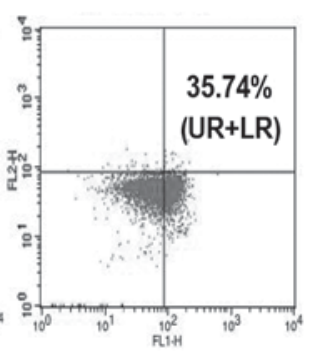

Annexin V

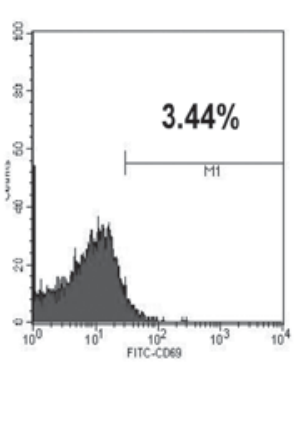

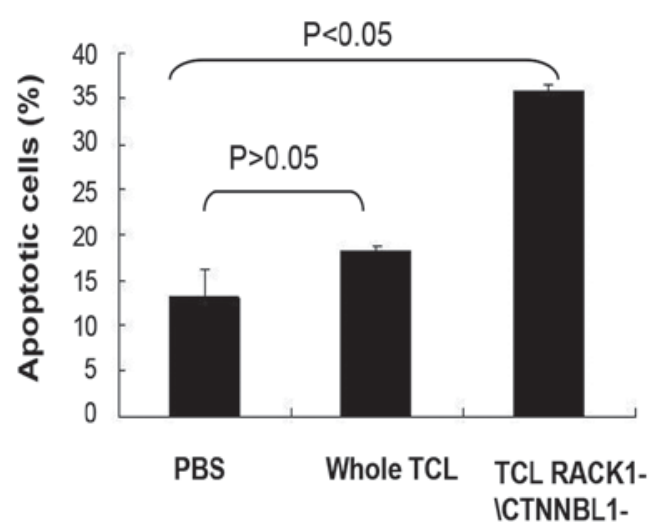

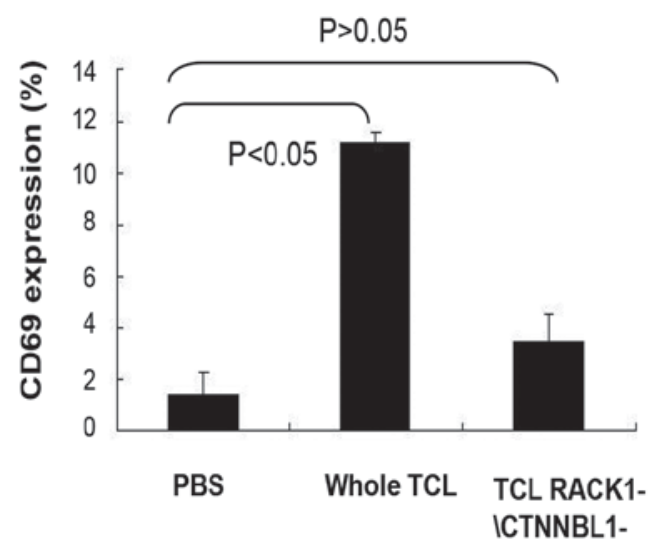

Figure 5. RACK1 and CTNNBL1-deficient TCL upregulates apoptosis and inhibits activation of mouse splenocytes. Mouse splenocytes were cultured for $48 \mathrm{~h}$ in medium containing PBS, whole TCL from Lewis lung cancer cells, or TCL lacking RACK1 and CTNNBL1. The rate of apoptosis and the expression of CD69 in splenocytes was determined by flow cytometry. (A) Numbers in each plot represent the percentage of Annexin V-positive cells (UR percentage + LR percentage). Representative data from one of three experiments is shown. Bar graphs indicate the percentage of apoptotic cells. Data are presented as the mean \pm standard deviation of three experiments. (B) Number in each histogram represents the CD69 mean fluorescence intensity of the sample. Representative data from one of the three experiments is shown. Bar graphs indicated the percentage of CD69-positive cells. Data are presented as the mean \pm standard deviation of three experiments. CD69, cluster of differentiation 69; CTNNBL1, catenin $\beta$-like 1; LR, lower right; PI, propidium iodide; RACK1, receptor for activated C kinase 1; TCL, tumor cell lysate; UR, upper right.

compared with in the control splenocytes stimulated with PBS; however, the result was not statistically significant $(\mathrm{P}>0.05$; Fig. 5A). In addition, the early activation marker CD69 was significantly upregulated in splenocytes stimulated with whole TCL ( $\mathrm{P}<0.05$; Fig. 5B). Conversely, CD69 expression in mouse splenocytes stimulated with TCL lacking RACK1 and CTNNBL1 was downregulated $(\mathrm{P}<0.05$; Fig. $5 \mathrm{~B})$, whereas Annexin $\mathrm{V}$ expression was upregulated in these cells $(\mathrm{P}>0.05$; Fig. 5A). These results indicated that RACK1 and CTNNBL1 may be the main elements in TCL that affect activation and apoptosis of mouse splenocytes.

\section{Discussion}

Activation of immunocytes has a key role in antitumor immunotherapy. Numerous studies have reported that TCL can activate immunocytes (22-24); however, it remains unclear as to what components present in TCL possess this ability. TCL is a protein mixture produced by lysed tumor cells; the majority of these proteins are involved in signaling pathways associated with cell division and activation. Therefore, it is hypothesized that TCL depends on these proteins to activate immunocytes. The present study identified the proteins in
Lewis lung cancer cell TCL using iTRAQ detection. Among the proteins, RACK1, CTNNBL1, Cullin 3, BCLAF1, RPS6, SOD1, HMGB1, ING1 and ERCC3 have been reported to activate cells and inhibit apoptosis, according to protein databases and previous studies $(8,9,15-21)$. Among these proteins, only RACK1, CTNNBL1 and Cullin 3 could be detected by western blotting, and the protein expression levels of RACK1 and CTNNBL1 in TCL were higher than the levels of Cullin 3. Therefore, RACK1 and CTNNBL1 may serve a more important role in immunocyte activation.

Pathways involved in cell activation and apoptosis in almost all animal cells require RACK1, a Sonic hedgehog signal pathway protein (8), and CTNNBL1, a Wnt signal pathway protein (9). Therefore, RACK1 and CTNNBL1 may be involved in the activation of non-immunocytes and immunocytes. RACK1 knockdown in monocytes has been reported to lead to impaired proliferation and decreased interleukin (IL)-6 and tumor necrosis factor (TNF)- $\alpha$ expression, thus indicating that RACK1 can activate monocytes (25). Conversely, to the best of our knowledge, there is no direct evidence to suggest that CTNNBL1 can activate immunocytes. However, B lymphocyte differentiation and antibody production require CTNNBL1 to stabilize activation-induced 
cytidine deaminase, which is a critical enzyme associated with B cell differentiation and antibody production (12). Therefore, the role of CTNNBL1 may be similar to RACK1, but with the ability to activate B lymphocytes, depending on its cell activation signaling pathway.

To investigate the role of these proteins in the TCL-induced activation of immunocytes, the proteins were removed from the TCL and the difference in TCL functionality was determined. RACK1 and CTNNBL1 were isolated from Lewis lung cancer TCL using an IP-based method. IP is used to separate individual proteins from cells to study the relationship between various proteins. In this process, the cell lysate should be prepared first, and then the protein of interest can be isolated using a specific antibody and collected using agarose beads to further analyze the protein function. Alternatively, to remove RACK1 and CTNNBL1, the unbound flow-through was collected after IP, rather than the RACK1 and CTNNBL1 isolate. In theory, three or more proteins can be removed from TCL by repeating this procedure, and this method may be of value in understanding TCL-based cancer immunotherapy.

Incubation with the RACK1 and CTNNBL1-deficient TCL induced apoptosis of mouse splenocytes; however, their activation was reduced compared with those stimulated by whole TCL. This finding indicated that RACK1 and CTNNBL1 in TCL serve a key role in mouse splenocyte activation. Mouse splenocytes consist of lymphocytes and monocytes. Among these cells, macrophages and DCs are able to ingest exogenous protein $(26,27)$. It has been reported that purified glutathione S-transferase can be ingested and transferred the cell membrane in RAW264.7 mouse macrophages, where it participates in signaling pathways associated with cell proliferation, apoptosis and cell division (28). Similarly, ovalbumin protein can be ingested by DCs as an exogenous protein, which may be used to estimate DC immunoactivity (29). B lymphocytes are also able to present antigens during the immune response (30). Based on these findings, it is hypothesized that RACK1 and CTNNBL1 may activate mouse splenocytes as follows: When TCL is cocultured with mouse splenocytes, RACK1 and CTNNBL1 may be obtained by monocytes or B lymphocytes, which are consequently activated. The activated monocytes or B lymphocytes possess a stronger antigen-presenting ability and secrete more IL- 6 and TNF- $\alpha$, which promotes activation of T and $B$ lymphocytes, and natural killer cells. In addition, expression of the early immunocyte marker, CD69, is increased in these mouse splenocytes. The present study suggested that RACK1 and CTNNBL1-induced activation of monocytes or B lymphocytes is a key stage in mouse splenocyte activation. In these cells, RACK1 and CTNNBL1, via cell signaling pathways, inhibit apoptosis and promote activation. With regards to the proliferation of non-immunocytes, RACK1 and CTNNBL1 function via different cell signaling pathways. RACK1 promotes tumor cell proliferation and inhibits apoptosis via the Sonic hedgehog signaling pathway (8), whereas CTNNBL1 enhances proliferation through the Wnt signaling pathway (9). Therefore, it may be hypothesized that RACK1 and CTNNBL1 activate the same signaling pathways to induce activation of mouse monocytes or B lymphocytes, and inhibit their apoptosis. However, this hypothesis requires further experimental verification, which we aim to pursue in future experiments. Furthermore, although low quantities were detected in the TCL in the present study, seven other proteins (CULLIN 3, BCLAF1, RPS6, SOD1, HMGB1, ING1 and ERCC3) may influence immunocyte activation or apoptosis; further studies are required to clarify their roles.

In conclusion, the present study identified a novel IP-based method for the removal of proteins from TCL. Using this method, it was demonstrated that RACK1 and CTNNBL1 are the key elements in Lewis lung cancer TCL, which are involved in the activation of mouse splenocytes. This approach may be used to generate TCL-based tumor vaccines for human use.

\section{Acknowledgements}

The present study was supported by the National Natural Science Foundation (grant no. 81402351), the 2015 Anhui Province Funded Project of Study Abroad (grant no. 5), and the Anhui Laboratory of Biological Macro-molecules Research Foundation (grant no. 1306C083008).

\section{References}

1. OginoS, Galon J, Fuchs CS and Dranoff G: Cancer immunology-analysis of host and tumor factors for personalized medicine. Nat Rev Clin Oncol 8: 711-719, 2011.

2. Borghaei H, Smith MR and Campbell KS: Immunotherapy of cancer. Eur J Pharmacol 625: 41-54, 2009.

3. Chiang CL, Coukos G and Kandalaft LE: Whole tumor antigen vaccines: Where are we? Vaccines (Basel) 3: 344-372, 2015.

4. González FE, Gleisner A, Falcón-Beas F, Osorio F, López MN and Salazar-Onfray F: Tumor cell lysates as immunogenic sources for cancer vaccine design. Hum Vaccin Immunother 10: 3261-3269, 2014

5. Son CH, Bae JH, Shin DY, Lee HR, Yang K and Park YS: Antitumor effect of dendritic cell loaded ex vivo and in vivo with tumor-associated antigens in lung cancermodel. Immunol Invest 43: 447-462, 2014.

6. Dong B, Sun L, Wu X, Zhang P, Wang L, Wei H, Zhou L, Hu X, Yu Y, Hua S and Wang L: Vaccination with TCL plus MHSP65 induces anti-lung cancer immunity in mice. Cancer Immunol Immunother 59: 899-908, 2010.

7. Li JJ and Xie D: RACK1, a versatile hub in cancer. Oncogene 34: 1890-1898, 2015

8. Shi S, Deng YZ, Zhao JS, Ji XD, Shi J, Feng YX, Li G, Li JJ, Zhu D, Koeffler HP, et al: RACK1 promotes non-small-cell lung cancer tumorigenicity through activating sonic hedgehog signaling pathway. J Biol Chem 287: 7845-7858, 2012.

9. Huhn S, Ingelfinger D, Bermejo JL, Bevier M, Pardini B, Naccarati A, Steinke V, Rahner N, Holinski-Feder E, Morak M, et al: Polymorphisms in CTNNBL1 in relation to colorectal cancer with evolutionary implications. Int J Mol Epidemiol Genet 2: 36-50, 2011.

10. van Maldegem F, Maslen S, Johnson CM, Chandra A, Ganesh K, Skehel M and Rada C: CTNNBL1 facilitates the association of CWC15 with CDC5L and is required to maintain the abundance of the Prp19 spliceosomal complex. Nucleic Acids Res 43: 7058-7069, 2015.

11. Ganesh K, Adam S, Taylor B, Simpson P, Rada C and Neuberger M: CTNNBL1 is a novel nuclear localization sequence-binding protein that recognizes RNA-splicing factors CDC5L and Prp31. J Biol Chem 286: 17091-17102, 2011.

12. Conticello SG, Ganesh K, Xue K, Lu M, Rada C and Neuberger MS: Interaction between antibody-diversification enzyme AID and spliceosome-associated factor CTNNBL1. Mol Cell 31: 474-484, 2008.

13. Livak KJ and Schmittgen TD: Analysis of relative gene expression data using real-time quantitative PCR and the 2(-Delta Delta C(T)) method. Methods 25: 402-408, 2001.

14. Svec D, Tichopad A, Novosadova V, Pfaffl MW and Kubista M: How good is a PCR efficiency estimate: Recommendations for precise and robust qPCR efficiency assessments. Biomol Detect Quantif 3: 9-16, 2015. 
15. Dorr C, Janik C, Weg M, Been RA, Bader J, Kang R, Ng B, Foran L, Landman SR, O'Sullivan MG, et al: Transposon mutagenesis screen identifies potential lung cancer drivers and CUL3 as a tumor suppressor. Mol Cancer Res 13: 1238-1247, 2015.

16. Zhou X, Li X, Cheng Y, Wu W, Xie Z, Xi Q, Han J, Wu G, Fang J and Feng Y: BCLAF1 and its splicing regulator SRSF10 regulate the tumorigenic potential of colon cancer cells. Nat Commun 5: 4581, 2014.

17. Knoll M, Macher-Goeppinger S, Kopitz J, Duensing S, Pahernik S, Hohenfellner M, Schirmacher P and Roth W. The ribosomal protein S6 in renal cell carcinoma: Functional relevance and potential as biomarker. Oncotarget 7: 418-432, 2016.

18. Skrzycki M, Czeczot H, Chrzanowska A and Otto-Slusarczyk D The level of superoxide dismutase expression in primary and metastatic colorectal cancer cells in hypoxia and tissue normoxia. Pol Merkur Lekarski 39: 281-286, 2015 (In Polish).

19. Sharma S, Evans A and Hemers E: Mesenchymal-epithelial signalling in tumour microenvironment: Role of high-mobility group Box 1. Cell Tissue Res 365: 357-366, 2016.

20. Han X, Chen Y, Yao N, Liu H and Wang Z: MicroRNA let-7b suppresses human gastric cancer malignancy by targeting ING1. Cancer Gene Ther 22: 122-129, 2015.

21. Terashita Y, Ishiguro H, Haruki N, Sugiura H, Tanaka T, Kimura M, Shinoda N, Kuwabara Y and Fujii Y. Excision repair cross complementing 3 expression is involved in patient prognosis and tumor progression in esophageal cancer. Oncol Rep 12: $827-831,2004$

22. Kawahara $\mathbf{M}$ and Takaku $\mathrm{H}$ : A tumor lysate is an effective vaccine antigen for the stimulation of CD4(+) T-cell function and subsequent induction of antitumor immunity mediated by CD8(+) T cells. Cancer Biol Ther 16: 1616-1625, 2015.
23. Gershan JA, Barr KM, Weber JJ, Jing W and Johnson BD: Immune modulating effects of cyclophosphamide and treatment with tumor lysate/CpG synergize to eliminate murine neuroblastoma. J Immunother Cancer 3: 24, 2015.

24. Yuan X, Li W, Cui Y, Zhan Q, Zhang C, Yang Z, Li X, Li S, Guan Q and Sun X: Specific cellular immune response elicited by the necrotic tumor cell-stimulated macrophages. Int Immunopharmacol 27: 171-176, 2015.

25. Zhang DL: The role of scaffold RACK1 in the production of proinflammatory cytokines [Dissertation]. Guang Zhou, Zhongnan University, 2013.

26. O'Neill LA and Pearce EJ: Immunometabolism governs dendritic cell and macrophage function. J Exp Med 213: 15-23, 2016.

27. Cybulsky MI, Cheong C and Robbins CS: Macrophages and dendritic cells: Partners in atherogenesis. Circ Res 118: 637-652, 2016.

28. Wang Y, Shen JY, Luo L and Yin ZM: The Location of FITC-GSTP1 in the Murine Macrophages. J Nanjing Norm Univ (Nat Sci Ed): 105-110, 2008.

29. Wirsdörfer F, Bangen JM, Pastille E, Schmitz D, Flohé S, Schumak B and Flohé SB: Dendritic cell-like cells accumulate in regenerating murine skeletal muscle after injury and boost adaptive immune responses only upon a microbial challenge. PLoS One 11: e0155870, 2016.

30. Wennhold K, Weber TM, Klein-Gonzalez N, Thelen M, Garcia-Marquez M, Chakupurakal G, Fiedler A, Schlösser HA, Fischer R, Theurich S, et al: CD40-activated B cells induce anti-tumor immunity in vivo. Oncotarget 8: 27740-27753, 2017. 